\title{
The Wigglez Galaxy Survey shows that dark energy is real
}

Michael DRINKWATER*

University of Queensland

E-mail: m.drinkwater@uq.edu.au

Chris BLAKE

Swinburne University

E-mail: cblakedastro.swin.edu.au

\section{Tamara DAVIS}

University of Queensland

E-mail: tamarad@physics.uq.edu.au

\section{and the WiggleZ Team}

http://wigglez.swin.edu.au

The WiggleZ Dark Energy Survey on the Anglo-Australian Telescope has measured redshifts for 220,000 emission line galaxies. The galaxies sample a volume of 1 cubic Gpc and a redshift range of $0.2<z<1$. This is the first survey to measure the cosmology of the universe over such a wide range of epochs. Our analysis provides strong evidence that dark energy is real.

In our first major results we made two successful tests of the standard (" $\Lambda \mathrm{CDM}$ ") cosmological model dominated by a cosmological constant $(\Lambda)$ and cold dark matter (CDM). First, we measured the effect of dark energy on the gravitational growth rate of cosmic structure. The measured growth rate is entirely consistent with the $\Lambda$ CDM model over the whole redshift range measured. Second, we detected the imprint of baryon acoustic oscillations in the clustering of the WiggleZ galaxies, allowing us to measure the cosmological distance-redshift relation at $z=0.6$. The results confirm the $\Lambda \mathrm{CDM}$ model, as well as providing evidence for accelerating expansion of the universe that is independent of earlier supernova measurements.

In a very different analysis of the WiggleZ data we used a novel method to make a direct, geometric measurement of the expansion rate of the Universe as a function of time. The increase in the expansion rate over the last 7 billion years shows that the universe is accelerating, independent of any cosmological model.

The 2011 Europhysics Conference on High Energy Physics-HEP 2011,

July 21-27, 2011

Grenoble, Rhône-Alpes France

\footnotetext{
${ }^{*}$ Speaker.
} 


\section{The WiggleZ Dark Energy Survey}

A major part of cosmology in recent decades has been the process of testing different cosmological models. A major advance was made in the 1990s when Type Ia supernovae were used as distance indicators to show that observations were clearly inconsistent with models of empty or decelerating (i.e. matter-dominated) universes $[1,2]$. The supernova data were better-fitted by accelerating models such as universes dominated by a cosmological constant. However they are also consistent with several models based on modified theories of gravity: other types of observation are needed to differentiate these models[3]

The WiggleZ Dark Energy Survey[4] was designed to use measurements of large-scale structure to provide a complementary approach. The survey has measured redshifts of some 220,000 galaxies in a volume of $1 \mathrm{Gpc}^{3}$, the largest volume ever surveyed at such high redshifts $(0.2<$ $z<1.0$ ). In this paper we present three key results from the project: two tests of the standard cosmological model and a model-independent confirmation of the acceleration of the Universe.

\section{High-redshift measurement of the growth of structure}

The large-scale distribution of galaxies can be used to measure the growth of structure over time due to gravitational clustering. This measurement can be used to test cosmological models that are not separated by the distance indicators described above. In Figure 1 we show the growth rate parameter measured as a function of redshift from the WiggleZ data[5] and earlier work. Our data are consistent with the standard $\Lambda \mathrm{CDM}$ model over the full redshift range of the survey.

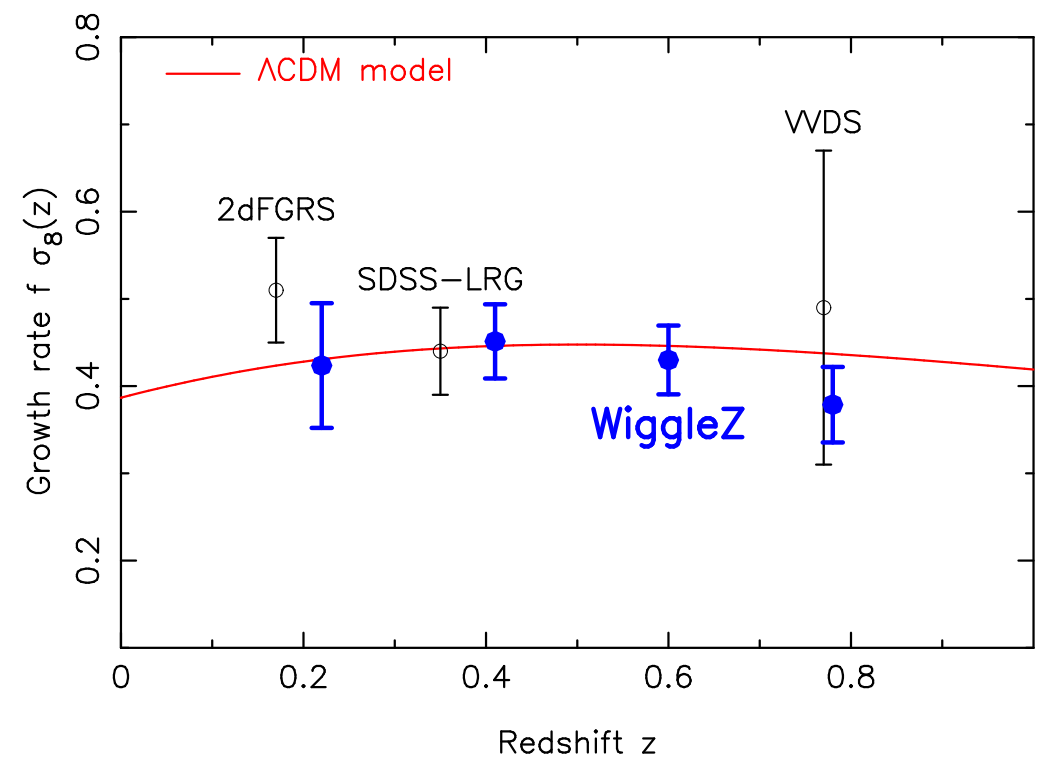

Figure 1: The growth rate of cosmic structure (parameterised by $f(z) \sigma_{8}(z)$ ) obtained in four redshift slices by fitting WiggleZ Survey data. The WiggleZ measurements are compared to results previously published for the 2dFGRS, SDSS-LRG, and VVDS samples (black open circles). The prediction of a flat $\Lambda$ CDM cosmological model is also shown. See [5] for details. 


\section{Geometric measurement of the expansion rate of the Universe}

As noted above the conclusion that the universe is accelerating was based on fitting a model in which possible acceleration was a parameter. With large-scale structure data we can now test for the acceleration in an entirely model-independent fashion. Sufficiently large structures can be assumed to be spherical, so we can take the ratio of their radial and tangential sizes and eliminate the cosmological model to derive a direct measure of the Hubble constant at that epoch [6]. Figure 2 shows this direct measurement of the expansion rate at 4 epochs, providing the first modelindependent evidence that the expansion rate is increasing, i.e. we live in an accelerating Universe.

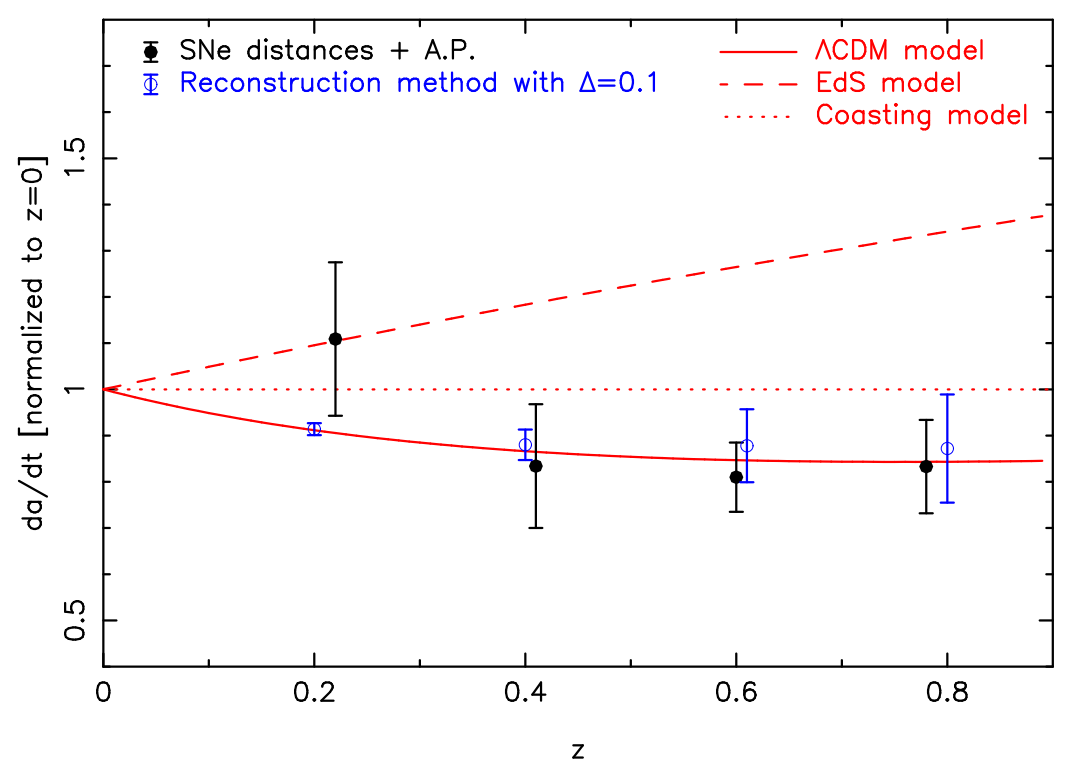

Figure 2: The change in the expansion rate of the Universe as a function of redshift. The expansion rate is normalised by the current $(z=0)$ rate; accelerating expansion implies a decrease in the value of this quantity with increasing redshift. Predictions are plotted for three different models: a fiducial CDM model with $\Omega_{M}=0.27$ (solid line), an Einstein de-Sitter model with $\Omega_{M}=1$ (dashed line), and a "coasting" model with a constant expansion rate (dotted line). See [6] for details.

\section{High-redshift measurement of baryon acoustic oscillations}

The main original aim of the WiggleZ project was to measure the angular size of the baryon acoustic oscillation (BAO) scale ${ }^{1}$ at a redshift of $z \approx 0.6$. We successfully detected this feature at three different redshifts [7] as shown in Fig. 3.

Combining our detection with previous low-redshift measurements we show that the BAO signal has been detected at significance of $5 \sigma$. The BAO results now measure the distance scale with a similar precision to the supernova results, as shown in Fig. 4. Our results provide an independent confirmation of the previous supernovae results and the combined results are consistent with a flat Universe with a cosmological constant.

\footnotetext{
${ }^{1}$ The BAO scale is essentially a standard ruler scale of size about $100 h^{-1} \mathrm{Mpc}$ in the large-scale distribution of galaxies.
} 

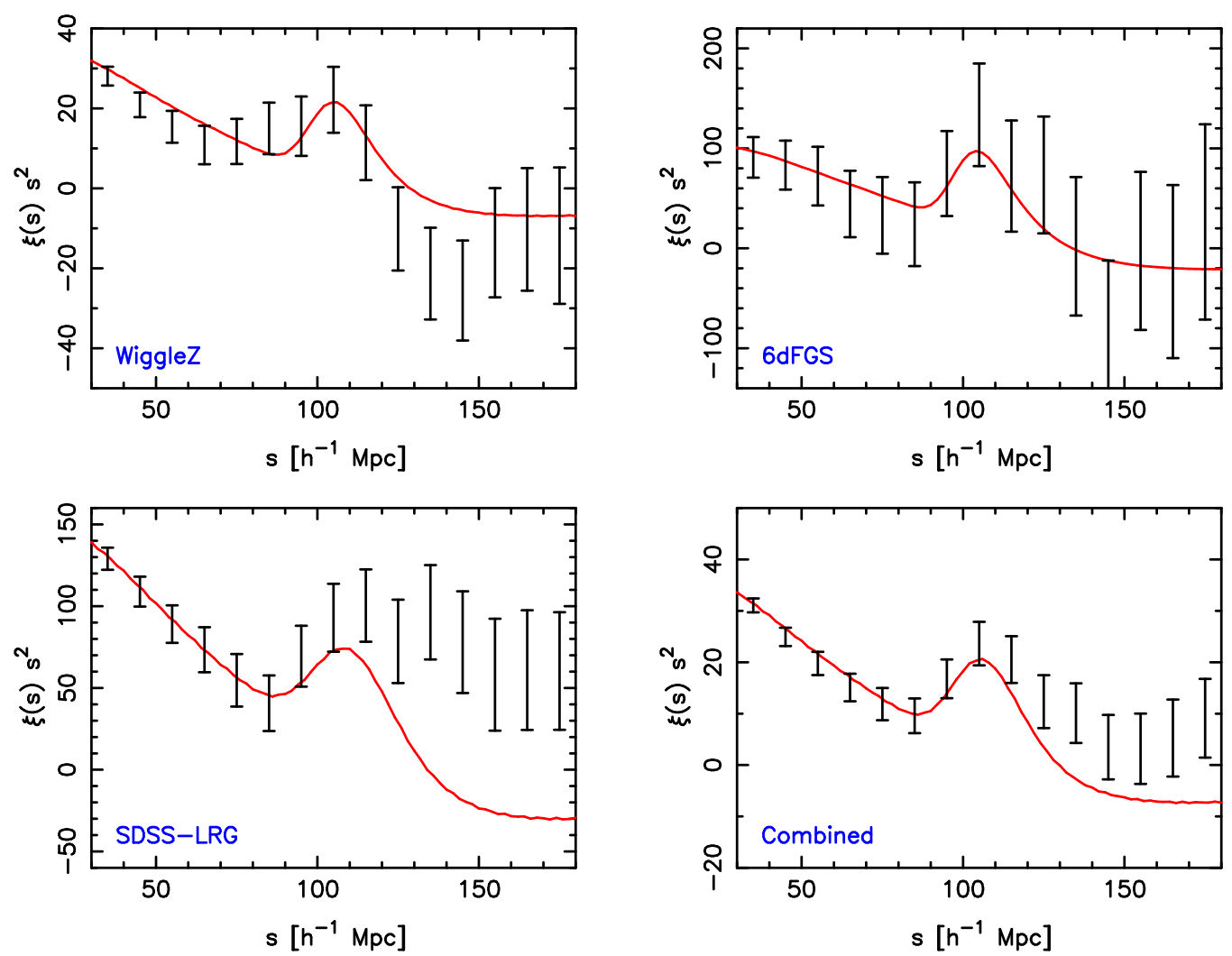

Figure 3: The galaxy correlation function $\xi(s)$, measured for various redshift ranges from the WiggleZ data, plotted as $\xi(s) s^{2}$ where $s$ is the co-moving redshift-space separation. The best-fitting clustering models in each case are overplotted as the solid lines. Significant detections of the baryon acoustic peak are obtained in each separate redshift slice[7].

\section{References}

[1] A. G. Riess et al., Observational Evidence from Supernovae for an Accelerating Universe and a Cosmological Constant, AJ, 116, 1009

[2] S. Perlmutter, et al., Measurements of Omega and Lambda from 42 High-Redshift Supernovae ApJ, 517,565

[3] T. Davis et al., Scrutinizing Exotic Cosmological Models Using ESSENCE Supernova Data Combined with Other Cosmological Probes, ApJ, 666, 716

[4] M. J. Drinkwater et al., The WiggleZ Dark Energy Survey: Survey Design and First Data Release, MNRAS, 401, 1429

[5] C. Blake et al., The WiggleZ Dark Energy Survey: the growth rate of cosmic structure since redshift $z$ = 0.9, MNRAS, 415, 2876

[6] C. Blake et al., The WiggleZ Dark Energy Survey: measuring the cosmic expansion history using the Alcock-Paczynski test and distant supernovae, MNRAS, in press [arXiv:1108.2637]

[7] C. Blake et al., The WiggleZ Dark Energy Survey: mapping the distance-redshift relation with baryon acoustic oscillations, MNRAS, in press, [arXiv:1108.2635] 


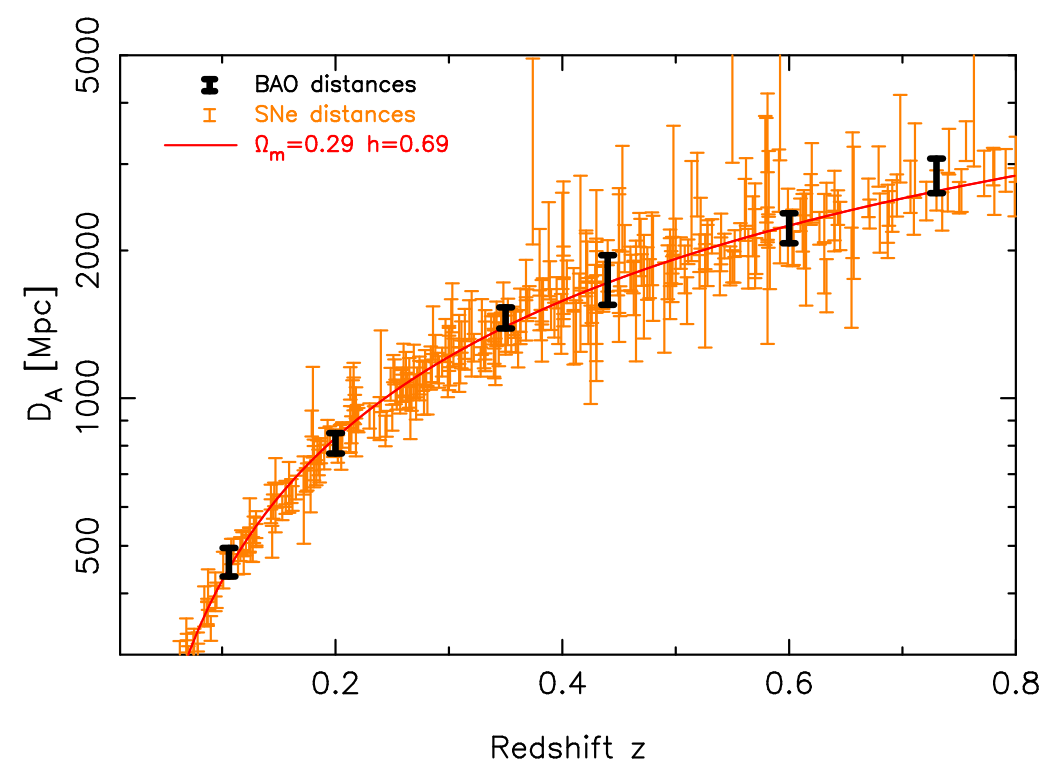

Figure 4: Comparison of the accuracy with which supernovae and baryon acoustic oscillations map out the cosmic distance scale at $z<0.8$ [7]. For the purposes of this Figure, BAO measurements of $D_{V}(z)$ have been converted into $D_{A}(z)$ assuming a Hubble parameter $H(z)$ for a flat $\Lambda C D M$ model, indicated by the solid line, and supernova measurements of $D_{L}(z)$ have been plotted assuming $D_{A}(z)=D_{L}(z) /(1+z)^{2}$. 